Toxicogenomics/proteomics Report

\title{
Inhibition of F-box protein FBXO6 gene expression by RNA interference enhances cadmium toxicity in HEK293 cells
}

\author{
Gi-Wook Hwang, Ke Du, Tsutomu Takahashi and Akira Naganuma \\ Laboratory of Molecular and Biochemical Toxicology, Graduate School of Pharmaceutical Sciences, \\ Tohoku University, Aoba-ku, Sendai, Miyagi 980-8578, Japan
}

(Received September 10, 2011; Accepted October 15, 2011)

\begin{abstract}
Cadmium is a typical environmental pollutant and the causative agent of itai-itai disease. Ubiquitin ligases potentially involved in mediating the effects of cadmium toxicity were investigated by RNA interference using a panel of small inhibitory (si)RNAs. The results demonstrated that the inhibition of gene expression of FBXO6 sensitizes HEK293 cells to cadmium.
\end{abstract}

Key words: Cadmium, Ubiquitin ligase, F-box protein, FBXO6

\section{INTRODUCTION}

The adverse effects of long-term exposure to trace amounts of cadmium in foods such as rice have become a public health problem (Satarug and Moore, 2004). Cadmium absorbed into the body is trapped by metallothionein, a protein that reduces heavy metal toxicity, thereby preventing or limiting toxicity (Klaassen et al., 2009). The accumulation of cadmium to levels at or above the body's threshold may cause renal tubular damage.

The ubiquitin-proteasome (UP) system is a selective proteolytic system that is preserved from yeast to humans. Ubiquitin is a low molecular weight protein that when attached to a target protein signals proteasomal degradation of the target protein (Hershko and Ciechanover, 1998). The process involves the recognition of the target protein by a ubiquitin ligase, which catalyzes the conjugation of ubiquitin to the target protein. Various ubiquitin ligases are present in cells and regulate the levels of their target proteins in response to various conditions and stimuli to maintain normal biological responses (Hatakeyama and Nakayama, 2003; Cardozo and Pagano, 2004). The UP system has been shown to play an important role in cadmium sensitivity (Stewart et al., 2003; Hwang et al., 2007, 2008; Uekusa et al., 2009; Tokumoto et al., 2011). However, the ubiquitin ligases involved in mediating the biological effects of cadmium toxicity remain unclear. Here, we used RNA interference to suppress the gene expression of ubiquitin ligases in HEK293 cells and assessed the effects on cadmium sensitivity. The results showed that the loss of expression of FBXO6 conferred increased sensitivity of HEK293 cells to cadmium.

\section{MATERIALS AND METHODS}

\section{Transfection conditions and cell viability assay}

The human embryonic kidney cell line, HEK293, was grown in Dulbecco's modified Eagle's medium (DMEM) supplemented with $10 \%$ heat-inactivated fetal bovine serum, $100 \mathrm{IU} / \mathrm{ml}$ penicillin, and $100 \mathrm{mg} / \mathrm{ml}$ streptomycin. Cultures were maintained at $37^{\circ} \mathrm{C}$ in a humidified $10 \% \mathrm{CO}_{2}$ atmosphere. Double-stranded small inhibitory (si)RNAs, each of which targeted approximately 100 ubiquitin ligases, were purchased from Qiagen (Germantown, MD, USA). Transfection of HEK293 cells with the siRNAs was carried out according to a detailed protocol from Qiagen. Briefly, $2.5 \mu \mathrm{l} /$ per well of siRNA mixture (final concentration, $10 \mathrm{nM}$ ) containing two different double-stranded siRNAs were spotted into the wells of a 96-well plate. A total of $0.75 \mu$ l of HiPerFect ${ }^{\circledR}$ transfection reagent (Qiagen) was diluted in $24.25 \mu 1$ of OptiMEM (Invitrogen, Carlsbad, CA, USA) and the diluted reagent was added to the prespotted siRNA. The plate was incubated for $10 \mathrm{~min}$ at room temperature to allow the formation of transfection complexes, after which $5 \times 10^{3}$ HEK293 cells in $100 \mu \mathrm{l}$ of medium were added to the wells. After incubation for $48 \mathrm{hr}, 40 \mu \mathrm{M}$ cadmium chloride, a concentration sufficient to inhibit the growth of normal cells by about $50 \%$, was added to the wells and then the cells were cultured for an additional $48 \mathrm{hr}$. Cell

Correspondence: Akira Naganuma (E-mail: naganuma@m.tohoku.ac.jp) 
viability was determined by staining with Alamer Blue (Biosource, Camarillo, CA, USA; Hwang et al., 2010).

\section{RNA isolation and quantitative real-time PCR}

Double-stranded siRNAs targeting the FBXO6 gene and an AllStar ${ }^{\circledR}$ negative control siRNA were purchased from Qiagen. The corresponding target sequences were as follows: FBXO6 siRNA No. 1, CCGGCCGGACATCGTGGTTAA; FBXO6 siRNA No. 2, ACCCGTGTGCTGAAGAGGATA. Forty-eight hr after transfection with control or FBXO6 siRNAs, cells (approximately $1 \times 10^{6}$ cells/well in 6-well plates) were rinsed well with PBS. Total RNA was isolated using a FastPure ${ }^{\circledR}$ RNA kit (Takara, Shiga, Japan) and first strand cDNA synthesis was performed using a PrimeScrip ${ }^{\circledR}$ RT reagent kit (Takara) in accordance with the manufacturer's protocol (Chen et al., 2009; Kawakami et al., 2009). Quantitative real-time PCR reactions were carried out using SYBR ${ }^{\circledR}$ Premix EX Taq (Takara). The oligonucleotide sequences of the primers used for PCR were as follows: sense, 5'-AGCCCCAAGATGACCAGGAAC-3', and antisense, 5'-ACCCAGACACAGGATGGACAG-3' for $F B X O 6$; and sense, 5'-GCACCGTCAAGGCTGAGAAC-3', and antisense, 5'-TGGTGAAGACGCCAGTGGA-3' for $G A P D H$. Changes in FBXO6 mRNA levels were determined based on standard curves after calibration of the assay.

\section{RESULTS AND DISCUSSION}

Using the Biobase Biological Database (www.biobase-international.com), about 100 proteins that functioned as ubiquitin ligases were identified. Two double-stranded siRNAs of different sequences targeting each of the genes encoding these ubiquitin ligases were simultaneously introduced into HEK293 cells. siRNAtreated cells were cultured for $48 \mathrm{hr}$ in the presence of $40 \mu \mathrm{M}$ cadmium chloride, a concentration that suppressed the growth of normal cells by about $50 \%$. In this manner, FBXO6 was identified as a ubiquitin ligase whose expression conferred resistance to cadmium, as the suppression of $F B X O 6$ expression by RNA interference conferred increased sensitivity of HEK 293 cells to cadmium. To confirm these effects, each double-stranded siRNA targeting FBXO6 (siRNA No. 1 and No. 2) was introduced separately into HEK293 cells. Compared to control cells, treatment with FBXO6 siRNA No. 1 resulted in markedly increased sensitivity to cadmium, while treatment with FBXO6 siRNA No. 2 increased cadmium sensitivity only slightly (Fig. 1A). Cells carrying both siRNAs were more sensitive to cadmium than those carrying only FBXO6
siRNA No. 2 (Fig. 1A), and these cells also had the lowest levels of FBXO6 mRNA (approximately 29\% of that seen in control cells). FBXO6 mRNA levels were approximately $38 \%$ and $50 \%$ of control levels in cells carrying FBXO6 siRNA No. 1 and FBXO6 siRNA No. 2, respectively (Fig. 1B). These results demonstrated that the FBXO6 ubiquitin ligase is involved in reducing cadmium toxicity. Inhibition of FBXO6 expression had only a slight impact on the sensitivity of cells to methylmercury, tributyltin, and arsenic trioxide. In contrast to cadmium, suppression of FBXO6 expression rendered cells highly resistant to inorganic mercury (Fig. 2). Thus, FBXO6 seems to be involved at least in part in reducing cadmium toxicity.

FBXO6 is a ubiquitin ligase involved in the endoplasmic reticulum-associated protein degradation (ERAD)
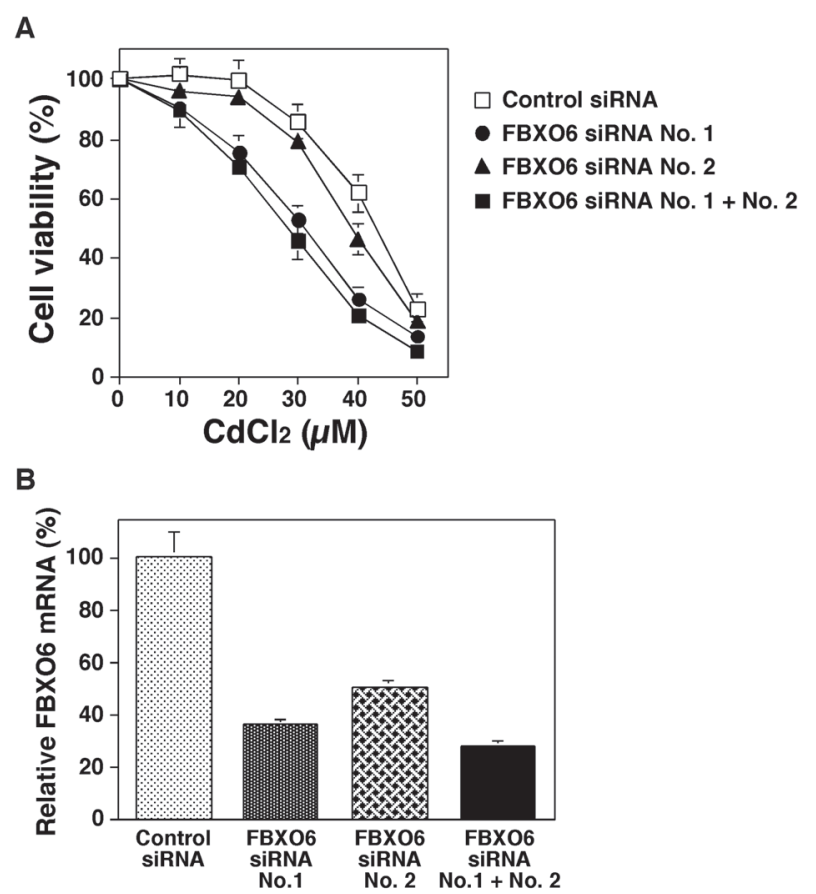

Fig. 1. Effect of gene knockdown of FBXO6 on the sensitivity of HEK293 cells to cadmium. (A) HEK293 cells $\left(5 \times 10^{3}\right.$ cells/well $)$ transfected with double-stranded FBXO6 siRNAs were seeded in 96-well plates and cultured in $100 \mu \mathrm{l}$ of DMEM. After incubation for $48 \mathrm{hr}$, cadmium chloride was added and cells were cultured for an additional $48 \mathrm{hr}$. Cell viability was determined by staining with Alamer Blue. Data represent the means and standard deviation (SD) of three cultures. (B) Levels of FBXO6 mRNA in HEK293 cells transfected with double-stranded FBXO6 siRNAs were analyzed by real-time PCR. The levels of FBXO6 mRNA were normalized to GAPDH mRNA. 
Down-regulation of FBXO6 by siRNA confers hypersensitivity to cadmium
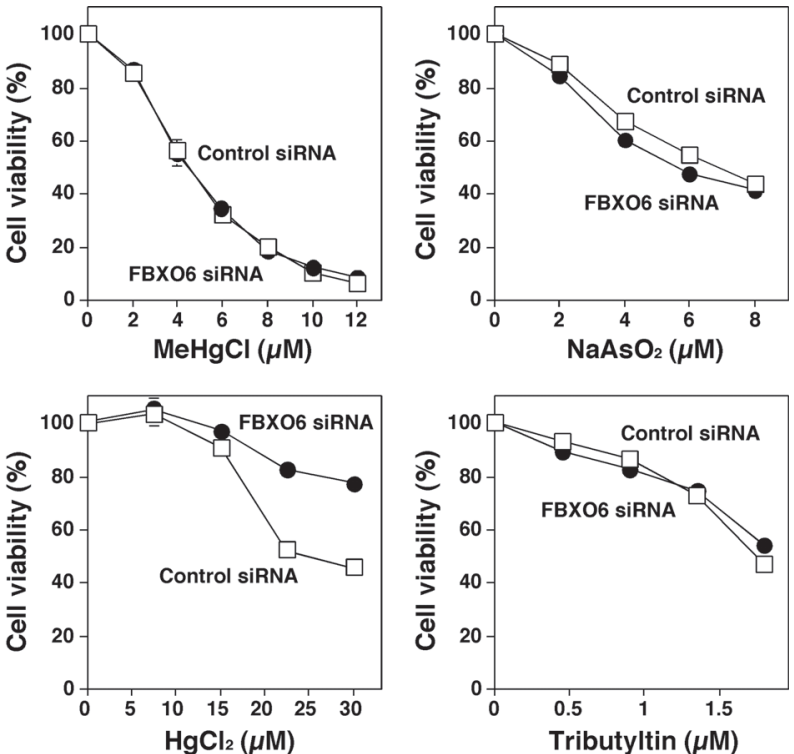

Fig. 2. Effect of gene knockdown of FBXO6 on the sensitivity of HEK293 cells to various metal compounds. HEK293 cells $\left(5 \times 10^{3}\right.$ cells/well $)$ transfected with double-stranded FBXO6 siRNAs were seeded in 96well plates and cultured in $100 \mu 1$ of DMEM. Metal treatment and the determination of cell viability were as described for Fig. 1A.

system and is responsible for the ubiquitination of N-glycoprotein (Yoshida et al., 2003). Endoplasmic reticulum (ER) stress has been reported to play a role in cadmium cytotoxicity (Kitamura and Hiramatsu, 2010). The current results build on these observations and suggest that FBXO6 may reduce ER stress caused by cadmium.

We have demonstrated here a novel role for the ubiquitin ligase FBXO6 in the reduction of cadmium toxicity. These results serve as a foundation for additional experiments exploring the mechanism(s) of cadmium toxicity, and specifically the role of FBXO6 in mediating the effects of cadmium toxicity.

\section{REFERENCES}

Cardozo, T. and Pagano, M. (2004): The SCF ubiquitin ligase: insights into a molecular machine. Nat. Rev. Mol. Cell. Biol., 5, 739-751.

Chen, Q., Wang, Z., Xiong, Y., Xue, W., Kao, X., Gao, Y., Muhammad, N. and Song, D. (2009): Selenium increases expression of HSP70 and antioxidant enzymes to lessen oxidative damage in Fincoal-type fluorosis. J. Toxicol. Sci., 34, 399-405.

Hatakeyama, S. and Nakayama, K.I. (2003): U-box proteins as a new family of ubiquitin ligases. Biochem. Biophys. Res. Commun., 302, 635-645.

Hershko, A. and Ciechanover, A. (1998): The ubiquitin system. Annu. Rev. Biochem., 67, 425-479.
Hwang, G.W., Furuchi, T. and Naganuma, A. (2007): Ubiquitinconjugating enzyme $\mathrm{Cdc} 34$ mediates cadmium resistance in budding yeast through ubiquitination of the transcription factor Met4. Biochem. Biophys. Res. Commun., 363, 873-878.

Hwang, G.W., Furuchi, T. and Naganuma, A. (2008): The ubiquitin-conjugating enzymes, Ubc4 and Cdc34, mediate cadmium resistance in budding yeast through different mechanisms. Life Sci., 82, 1182-1185.

Hwang, G.W., Tobita, M., Takahashi, T., Kuge, S., Kita, K. and Naganuma, A. (2010): siRNA-mediated AMPK $\alpha 1$ subunit gene PRKAA1 silencing enhances methylmercury toxicity in HEK293 cells. J. Toxicol. Sci., 35, 601-604.

Kawakami, T., Ito, T., Ohsako, S., Shiizaki, K., Murakami, Y., Hirowatari, K., Sato, M. and Tohyama, C. (2009): Possible involvement of arylhydrocarbon receptor variants in TCDDinduced thymic atrophy and XRE-dependent transcriptional activity in Wistar Hannover GALAS rats. J. Toxicol. Sci., 34, 209-220.

Kitamura, M. and Hiramatsu, N. (2010): The oxidative stress: endoplasmic reticulum stress axis in cadmium toxicity. Biometals, 23, 941-950.

Klaassen, C.D., Liu, J. and Diwan, B.A. (2009): Metallothionein protection of cadmium toxicity. Toxicol. Appl. Pharmacol., 238, 215-220.

Satarug, S. and Moore, M.R. (2004): Adverse health effects of chronic exposure to low-level cadmium in foodstuffs and cigarette smoke. Environ. Health Perspect., 112, 1099-1103.

Stewart, D., Killeen, E., Naquin, R., Alam, S. and Alam, J. (2003): Degradation of transcription factor Nrf2 via the ubiquitin-proteasome pathway and stabilization by cadmium. J. Biol. Chem., 278, 2396-2402.

Tokumoto, M., Fujiwara, Y., Shimada, A., Hasegawa, T., Seko, Y., Nagase, H. and Satoh, M. (2011): Cadmium toxicity is caused by accumulation of p53 through the down-regulation of Ube2d family genes in vitro and in vivo. J. Toxicol. Sci., 36, 191-200.

Uekusa, H., Namimatsu, M., Hiwatashi, Y., Akimoto, T., Nishida, T., Takahashi, S. and Takahashi, Y. (2009): Cadmium interferes with the degradation of ATF5 via a post-ubiquitination step of the proteasome degradation pathway. Biochem. Biophys. Res. Commun., 380, 673-678.

Yoshida, Y., Tokunaga, F., Chiba, T., Iwai, K., Tanaka, K. and Tai, T. (2003): Fbs2 is a new member of the E3 ubiquitin ligase family that recognizes sugar chains. J. Biol. Chem., 278, 43877-43884. 